\title{
Wall Shear Stress Prediction Using Computational Simulation on Patient Specific Artery with Aneurysm
}

\author{
Muhamad Yunus ${ }^{1, \mathrm{a}}$, Anis Suhaila Shuib ${ }^{2, \mathrm{~b}}$, Tuan Mohammad Yusoff Shah ${ }^{1, \mathrm{c}}$, Ku Zilati Ku \\ Shaari $^{3, \mathrm{~d}}$, and Ahmad Sobri Muda ${ }^{4, \mathrm{e}}$ \\ ${ }^{1}$ Mechanical Engineering Department, Universiti Teknologi PETRONAS Malaysia \\ ${ }^{2}$ Chemical Engineering Department, Center for Intelligent Signal \& Imaging Research (CISIR), \\ Universiti Teknologi PETRONAS Malaysia \\ ${ }^{3}$ Chemical Engineering Department, Universiti Teknologi PETRONAS Malaysia \\ ${ }^{4}$ Radiology Department, Universiti Kebangsaan Malaysia Medical Centre (UKMMC)
}

\begin{abstract}
An aneurysm is formed when a blood vessel becomes dilated or distorted. It will cause the vessel to expand to a size greater than its original diameter. In this study, Wall Shear Stress (WSS) of cerebral artery with aneurysm was predicted using Computational Fluid Dynamics (CFD). WSS in the artery is one of the indicators for brain artery disease progression. Based on the results, the maximum value of blood velocity and WSS on patient specific artery with aneurysm are $3.23 \mathrm{~m} / \mathrm{s}$ and $60.1 \mathrm{~Pa}$, respectively. The location of high WSS is before and after the aneurysm bulge. The WSS is above the normal physiological value where the artery wall is exposed to high stress. Hence, the vessel at this location is anticipated to become weaker and could be further dilated.
\end{abstract}

\section{Introduction}

Aneurysm is a cerebrovascular disease which is related to disorder in the blood vessels of brain. The condition could lead to stroke and ischaemic attack. An aneurysm is formed when a blood vessel becomes dilated or distorted causing the vessel to expand to a size greater than its original diameter. Aneurysms can occur in a variety of blood vessels though they are most commonly found in the intracranial arteries. An aneurysm is a localized, blood-filled balloon-like bulge in the wall of a blood vessels. When the size of an aneurysm increases, there is a significant risk of rupture, resulting in severe bleeding, other complications or death. The mortality rate is almost $57 \%$ [1] for rupture occuring at a medical facility. Aneurysms can be hereditary or caused by disease, both of which cause the wall of the blood vessel to weaken [2]. Wall shear stress (WSS) has been proposed as a key parameter to characterize aneurysm [3]. Measurements of wall shear stress can help predict the areas at risk where aneurysms can rupture and this result is useful for clinical diagnosis data. This information assists medical practitioners in making clinical decision on the appropriate treatment for aneurysm.

\footnotetext{
ayunus_g02031@utp.edu.my, ${ }^{b}$ anisuha@petronas.com.my, ${ }^{c}$ tyusoff.ty@petronas.com.my, dkuzilati_kushaari@petronas.com.my, ${ }^{\mathrm{e}}$ sobri_muda@yahoo.com
} 
In recent years, many computational fluid dynamics (CFD) models have been constructed to study cerebral aneurysms [4]. CFD has the potential to be a useful clinical tool in observing vascular flow and disease pathology. In CFD, hemodynamic studies employed Newtonian $[3,5]$ and non-Newtonian models [6]. For studies which assume blood is a Newtonian fluid, the viscosity is taken as a constant value, thus ignoring the shear-thinning behavior of blood.

The objective of this work is to fundamentally understand the fluid dynamics factors that affect the aneurysm progression by simulating the velocity magnitude and wall shear stress in a section of artery with aneurysm. The basis of WSS value is $1.5 \mathrm{~Pa}$, which reported as a physiological value for cerebral artery [7].

\section{Methodology}

\subsection{Aneurysm Geometry}

Computational method is used where MIMICS 16.0 and ANSYS 14.5 software has been employed as a tool to mesh and segment image from Angiogram. A schematic of the patient specific artery is shown in Fig.1.

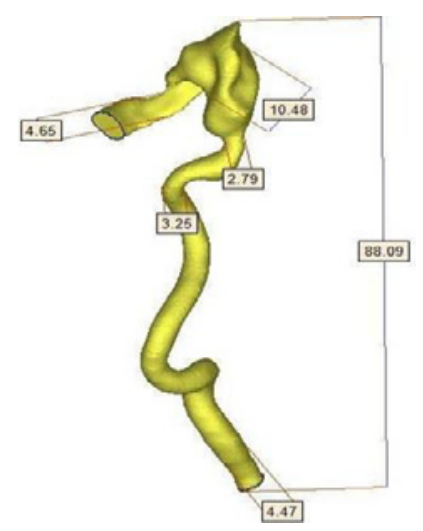

Figure 1. Schematic of the patient specific artery of varying diameter (in $\mathrm{mm}$ )

\subsection{Simulation model}

The governing Navier-Stokes equation can be expressed in a vector form as [8]:

$$
\rho \frac{D V}{D t}=-\Delta p+\mu \nabla^{2} \mathbf{V}+\rho S
$$

where $V$ is velocity magnitude, $\mu$ is viscocity of the fluid, $\rho$ is density of the fluid, and $S$ is body acting forces. The aneurysm diameter size is much larger than the size of the blood cells $\sim 5 \mu \mathrm{m}$, hence, the blood can be modeled as a continuum.

Blood is generally accepted as an incompressible fluid with a fluid density, $\rho=1080 \mathrm{~kg} / \mathrm{m}^{3}$. The vascular wall was treated as a rigid wall. Newtonian blood viscosity is taken as, $\mu=0.0041$ $\mathrm{kg} / \mathrm{m}-\mathrm{s}$ [9]. In order to calculate the WSS, the following relationship is used [10]:

$$
\tau_{\mathrm{w}}=-\mu \gamma=-\mu \frac{\partial v}{\partial r}
$$

where $\tau_{\mathrm{w}}$ is the wall shear stress, $\mu$ is the viscocity of blood and $\gamma$ is the shear rate. 
The inlet velocity which is in the form of a pulse flow is shown in Fig. 2 . The inlet flow is the average of velocity in the middle cerebral artery of 355 patients [11]. The pulse was set to $1 \mathrm{~s}$ and the results are captured at $0.25 \mathrm{~s}$ interval.

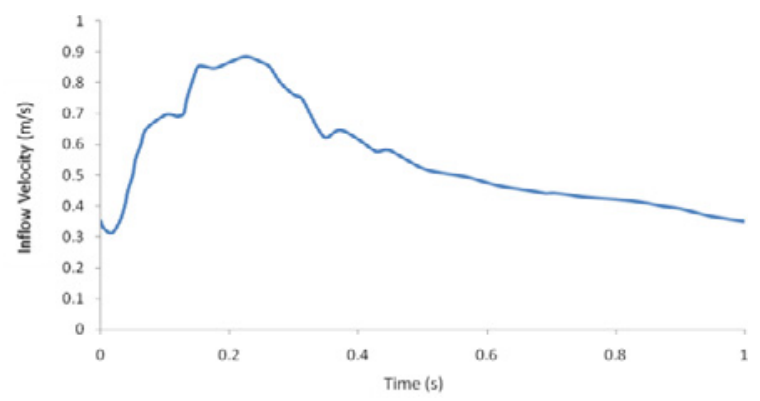

Figure 2. The inflow velocity profile in the artery with respect to time. [11]

\section{Results and Discussion}

The results of velocity and WSS was analyzed at time $t=0.25 \mathrm{~s}, 0.5 \mathrm{~s}, 0.75 \mathrm{~s}$ and $1 \mathrm{~s}$. The pulse is maximum at $\mathrm{t}=0.20 \mathrm{~s}$. As shown in equation 2 , velocity as an important parameter in obtaining the WSS value. The velocity pathline obtained for each time interval are plotted in Fig. 3 and contour of WSS is shown in Fig. 4. Maximum number for velocity pathline and WSS recorded $3.23 \mathrm{~m} / \mathrm{s}$ and $60.1 \mathrm{~Pa}$ respectively.
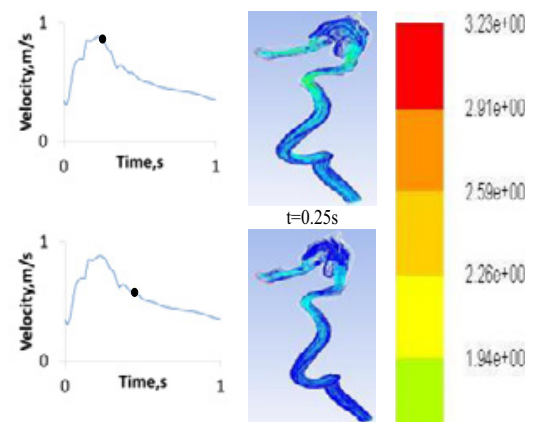

$2260+00$
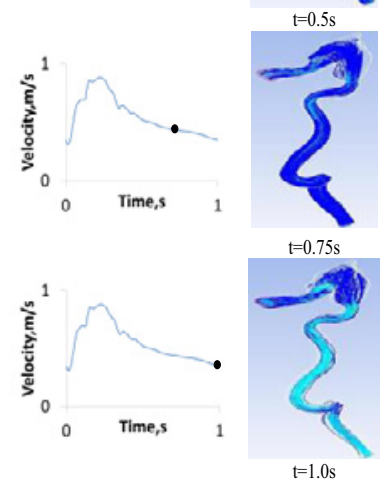

$1.94+00$

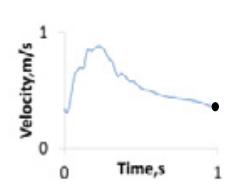

$t=1.0 \mathrm{~s}$

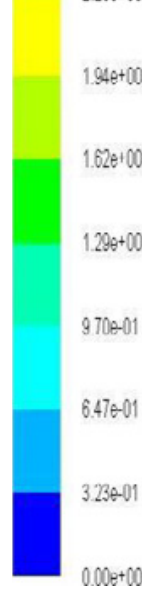

Figure 3. Velocity pathline for Newtonian model. 


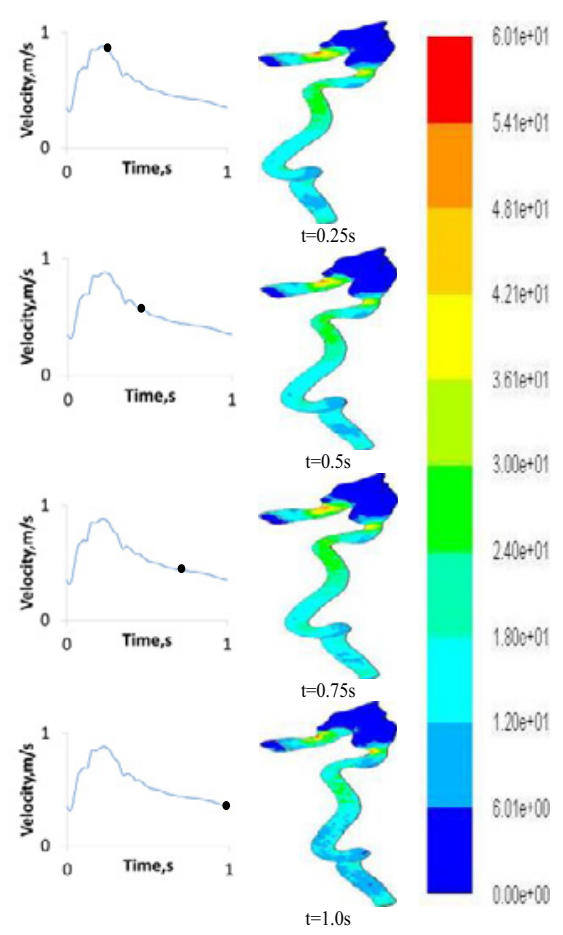

Figure 4. Wall Shear Stress for Newtonian model.

The model has a peak value in WSS of $60.1 \mathrm{~Pa}$ at $\mathrm{t}=0.25 \mathrm{~s}$ which can be observed in Fig. $4 \mathrm{a}$. The location of peak WSS is found prior to aneurysm bulge. The WSS decrease as the velocity decrease from peak pulse to $\mathrm{t}=1 \mathrm{~s}$. The WSS in the anuerysm bulge is the lowest due the sudden increase in area and corresponding reduction in the flow velocity.

\section{Conclusions}

Computational simulation is performed to predict the velocity in aneurysm bulge. Recirculation and reduction in flow velocity was observed in the aneurysm bulge. WSS in the bulge is within the physiological value. The risk of rupture is low. Maximum WSS which is above normal physiological value was observed immediately before and after the aneurysm. High flow rate impose increase stress to the artery wall.

\section{Acknowledgement}

The main author would like to express his gratitude to Universiti Teknologi PETRONAS for supporting and sponsoring the research.

\section{References}

1. M. J. Brown, A. J. Sutton, P. R. Bell, R. D. Sayers., A Meta Analysis of 50 Years of Ruptured Abdominal Aortic Aneurysm Repair. Br J Surg (2002). 89. p: 714-730. 
2. C. Basciano, C. Kleinstreuer, S. Hyun, and E. A. Finol., A Relation Between Near-Wall Particle-Hemodynamics and Onset of Thrombus Formation in Abdominal Aortic Aneurysms. Annals of Biomedics Engineering, 2011. 39(7): p. 2010-2026.

3. J. R. Blake, W. J. Easson, and P. R. Hoskins., A Dual Phantom System for Validation of Velocity Measurements in Stenosis Models Under Steady Flow. Elsevier Journal Ultrasound in Medical and Biology. 2009. 35(9): p. 1510-1524.

4. Harvey Ho, Jian Wu and P. Hunter., Blood Flow Simulation in a Giant Intracranial Aneurysm and Its Validation by Digital Subtraction Angiography. Springer science. 2011.

5. B. Luo, X. Yang, S. Wang, H. Li, J. Chen, H. Yu, Y. Zhang, Y. Zhang, S. Mu, Z. Liu and G. Ding., High Shear Stress and Flow Velocity in Partially Occluded Aneurysms Prone to Recanalization. Journal of American Heart Association, 2011. 42: p. 745-753.

6. V. L. Rayz, L. Boussel, M. T. Lawton, G. Acevedo-Bolton, L. Ge, W. L. Young, R. T. Higashida and D. Saloner., Numerical Modelling of the Flow in Intracranial Aneurysms : Prediction of Regions Prone to Thrombus Formation . Journal of Biomedical Engineering, 2008. 36: p. 1793-1804.

7. K. Kono, N. Tomura, R. Yoshimura, and T. Terada, "Changes in wall shear stress magnitude after aneurysm rupture," Acta Neurochir, vol. 155, pp. 1559-1563, 2013.

8. G. J. Sheard., Flow Dynamics and Wall Shear Stress Variation in a Fusiform Aneurysm. Journal Eng Math (2009). 64. p: 379-390.

9. S. O' Callaghan, M. Walsh and T. McGloughlin., Numerical modelling of Newtonian and nonNewtonian representation of blood in a distal end-to-side vascular bypass graft anastomosis. science direct elsevier 2006. 28: p. 70-74.

10. P. R. Hoskins and D. Hardman., three-dimensional imaging and computational modelling for estimation of wall stresses in arteries. British Journal of Radiology, 2009. 82: p. S3S17.

11. K. V. Sendstad, K. A. Mardal, M. Mortensen, B. A. P. Reif and H. P. Langtangen., Direct Numerical Simulation of Transitional Flow in a Patient-Spesific Intracranial Aneurysm. Journal of Biomechanics Elsevier, 2011. 44: p. 2826-2832. 